\title{
Einstein-Weyl structures on almost cosymplectic manifolds
}

\author{
Xiaomin Chen ${ }^{1}$ \\ Published online: 17 January 2019 \\ (c) The Author(s) 2019
}

\begin{abstract}
In this article, we study Einstein-Weyl structures on almost cosymplectic manifolds. First we prove that an almost cosymplectic $(\kappa, \mu)$-manifold is Einstein or cosymplectic if it admits a closed Einstein-Weyl structure or two Einstein-Weyl structures. Next for a three dimensional compact almost $\alpha$-cosymplectic manifold admitting closed Einstein-Weyl structures, we prove that it is Ricc-flat. Further, we show that an almost $\alpha$-cosymplectic admitting two Einstein-Weyl structures is either Einstein or $\alpha$-cosymplectic, provided that its Ricci tensor is commuting. Finally, we prove that a compact $K$-cosymplectic manifold with a closed Einstein-Weyl structure or two special Einstein-Weyl structures is cosymplectic.
\end{abstract}

Keywords Einstein-Weyl structures $\cdot$ Almost cosymplectic $(\kappa, \mu)$-manifolds $\cdot$ Almost $\alpha$-cosymplectic manifolds · Cosymplectic manifolds · Einstein manifolds

Mathematics Subject Classification 53D10 · 53D15

\section{Introduction}

A Weyl structure $W=(D,[g])$ on a smooth manifold $M$ is a torsion free affine connection $D$ preserving a conformal structure $[g]$. Namely there exists a unique 1-form $\theta$ such that $D g=-2 \theta \otimes g$. The concept of Weyl structure goes back to the work of Weyl. He introduced the definition to unify gravitational fields and electromagnetic fields (see [27]). Later on Hitchin [16] in studying 3-dimensional minitwistor theory observed that the minitwistor theory can be generalized over a 3-manifold endowed with a Weyl structure satisfying a certain Ricci tensor condition, called an Einstein-Weyl structure. Refer also to [17]. A Weyl structure $W=(D,[g])$ is Einstein-Weyl if the symmetrized Ricci tensor is proportional to a metric $g$ representing $[g]$ :

$$
\operatorname{Ric}^{D}(Y, X)+\operatorname{Ric}^{D}(X, Y)=\Lambda g(Y, X), \quad \Lambda \in C^{\infty}(M) .
$$

The author is supported by the Science Foundation of China University of Petroleum-Beijing (No. 2462015YQ0604), Natural Science Foundation of Beijing, China (Grant No.1194025) and partially supported by the Personnel Training and Academic Development Fund (No. 2462015QZDX02).

$\varangle \quad$ Xiaomin Chen

xmchen@cup.edu.cn

1 College of Science, China University of Petroleum (Beijing), Beijing 102249, China 
Further, if the unique 1-form $\theta$ is closed, then $W$ is said to be a closed Einstein-Weyl structure. The Einstein-Weyl condition plays a key role in physics, the pure Einstein theory being too strong as a system model for various physical questions. On the contrary, Einstein-Weyl structures appear naturally as the background of the static Yang-Mills-Higgs theory.

On the other hand, almost contact geometry also provides a natural underlying structure to analyse many problems in physics. For example, Sasakian-Einstein and 3-Sasakian geometry have emerged in the context of dualities of certain supersymmetric conformal field theories [3], and general almost contact structures have also been used to study special magnetic fields [26]. Meanwhile, Matzeu proved that several classes of almost contact manifolds also naturally carry Einstein-Weyl structures [19]. Therefore, Einstein-Weyl structures have received a lot of attentions in the frame work of almost contact metric manifold (see [11,20-22]).

Notice that an Einstein-Weyl structure is a generalization of Einstein metric in terms of affine connection. Recall the Goldberg conjecture [13] that a compact Einstein almost Kähler manifold is Kähler. The conjecture is true if the scalar curvature is non-negative [25]. As is well known, contact metric manifold can be considered as an odd-dimensional analogue to almost Kählar manifold. Boyer and Galicki [2] proposed an odd-dimensional Goldberg conjecture that a compact Einstein $K$-contact manifold is Sasakian and proved that it is true. As a generalization, Ghosh [12] and Gauduchon and Moroianu [10] simultaneously showed that a compact closed Einstein-Weyl $K$-contact manifold is also Sasakian using different method.

We also remark that another class of almost contact manifold, called almost cosymplectic manifold, was also paid many attentions (see a survey [5]). The concept was first defined by Goldberg and Yano [14] as an almost contact manifold whose 1-form $\eta$ and fundamental 2 -form $\omega$ are closed. An almost cosymplectic manifold is said to be cosymplectic if in addition the almost contact structure is normal (notice that here we adopt "cosymplectic" to represent "coKähler" in [5]). Concerning cosymplectic geometry, we mention the result that locally conformal cosymplectic manifolds admit a naturally defined conformally invariant Weyl structure [20]. Later on, Matzeu proved that every $(2 n+1)$-dimensional cosymplectic manifold of constant $\phi$-sectional curvature $c>0$ admits two Ricci-flat Weyl structures where the 1 -forms associated to the metric $g \in[g]$ are $\pm \theta= \pm \lambda \eta$, where $\lambda=\frac{2 c}{2 n-1}$. More recently, she generalized this result by proving that if a compact cosymplectic manifold $(M, \phi, \xi, \eta, g)$ admits a closed Einstein-Weyl structure $D$, then $M$ is necessarily $\eta$-Einstein [21].

Recently, Bazzoni and Goertsches [2] defined a $K$-cosymplectic manifold, namely an almost cosymplectic manifold whose Reeb vector field is Killing. In [4], in fact it is proved that every compact Einstein $K$-cosymplectic manifold is necessarily cosymplectic. In addition, Endo [8] defined the notion of almost cosymplectic $(\kappa, \mu)$-manifold, i.e., the curvature tensor of an almost cosymplectic manifold satisfies

$$
R(X, Y) \xi=\kappa(\eta(Y) X-\eta(X) Y)+\mu(\eta(Y) h X-\eta(X) h Y)
$$

for any vector fields $X, Y$, where $\kappa, \mu$ are constant and $h=\frac{1}{2} \mathcal{L}_{\xi} \phi$. As the extension of almost cosymplectic manifold, Kenmotsu [18] defined the almost Kenmotsu manifold, which is an almost contact manifold satisfying $d \eta=0$ and $d \omega=2 \eta \wedge \omega$. Based on this Kim and Pak [6] introduced the concept of almost $\alpha$-cosymplectic manifold, i.e., an almost contact manifold satisfying $d \eta=0$ and $d \omega=2 \alpha \eta \wedge \omega$ for some real number $\alpha$.

Motivated by the above background, in the present paper we first study an almost cosymplectic $(\kappa, \mu)$-manifold and an almost $\alpha$-cosymplectic manifold admitting Einstein-Weyl structures. Finally, we consider a compact $K$-cosymplectic manifold admitting a closed Einstein-Weyl structure. In order to prove our results, we need to recall some definitions and 
related conclusions on almost cosymplectic manifolds as well as Weyl structures, which are presented in Sects. 2 and 3, respectively. Starting from Sect. 4, we will state our results and give their proofs.

\section{Almost cosymplectic manifolds}

Let $M^{2 n+1}$ be a $(2 n+1)$-dimensional smooth manifold. An almost contact structure on $M$ is a triple $(\phi, \xi, \eta)$, where $\phi$ is a $(1,1)$-tensor field, $\xi$ a unit vector field, called Reeb vector field, $\eta$ a one-form dual to $\xi$ satisfying $\phi^{2}=-I+\eta \otimes \xi, \eta \circ \phi=0, \phi \circ \xi=0$. A smooth manifold with such a structure is called an almost contact manifold.

A Riemannian metric $g$ on $M$ is called compatible with the almost contact structure if

$$
g(\phi X, \phi Y)=g(X, Y)-\eta(X) \eta(Y), \quad g(X, \xi)=\eta(X)
$$

for any $X, Y \in \mathfrak{X}(M)$. An almost contact structure together with a compatible metric is called an almost contact metric structure and $(M, \phi, \xi, \eta, g)$ is called an almost contact metric manifold. An almost contact structure $(\phi, \xi, \eta)$ is said to be normal if the corresponding complex structure $J$ on $M \times \mathbb{R}$ is integrable.

Denote by $\omega$ the fundamental 2-form on $M$ defined by $\omega(X, Y):=g(\phi X, Y)$ for all $X, Y \in \mathfrak{X}(M)$. An almost $\alpha$-cosymplectic manifold $[6,24]$ is an almost contact metric manifold $(M, \phi, \xi, \eta, g)$ such that the fundamental form $\omega$ and 1-form $\eta$ satisfy $d \eta=0$ and $d \omega=2 \alpha \eta \wedge \omega$, where $\alpha$ is a real number. A normal almost $\alpha$-cosymplectic manifold is called $\alpha$-cosymplectic manifold. $M$ is an almost cosymplectic manifold if $\alpha=0$ and an almost Kenmotsu manifold if $\alpha=1$.

Let $M$ be an almost $\alpha$-cosymplectic manifold, we recall that there is an operator $h=\frac{1}{2} \mathcal{L}_{\xi} \phi$ which is a self-dual operator. The Levi-Civita connection is given by (see [24])

$$
2 g\left(\left(\nabla_{X} \phi\right) Y, Z\right)=2 \alpha g(g(\phi X, Y) \xi-\eta(Y) \phi X, Z)+g(N(Y, Z), \phi X)
$$

for arbitrary vector fields $X, Y$, where $N$ is the Nijenhuis torsion of $M$. Then by a simple calculation, we have

$$
\operatorname{trace}(h)=0, \quad h \xi=0, \quad \phi h=-h \phi, \quad g(h X, Y)=g(X, h Y), \quad \forall X, Y \in \mathfrak{X}(M) .
$$

Using (2.1), a straightforward calculation gives

$$
\nabla_{X} \xi=-\alpha \phi^{2} X-\phi h X
$$

and $\nabla_{\xi} \phi=0$. Denote by $R$ and Ric the Riemannian curvature tensor and Ricci tensor, respectively. For an almost $\alpha$-cosymplectic manifold $\left(M^{2 n+1}, \phi, \xi, \eta, g\right)$ the following equations were proved [24]:

$$
\begin{aligned}
& R(X, \xi) \xi-\phi R(\phi X, \xi) \xi=2\left[\alpha^{2} \phi^{2} X-h^{2} X\right], \\
& \left(\nabla_{\xi} h\right) X=-\phi R(X, \xi) \xi-\alpha^{2} \phi X-2 \alpha h X-\phi h^{2} X, \\
& \operatorname{Ric}(\xi, \xi)=-2 n \alpha^{2}-\operatorname{trace}\left(h^{2}\right), \\
& \operatorname{trace}(\phi h)=0, \\
& R(X, \xi) \xi=\alpha^{2} \phi^{2} X+2 \alpha \phi h X-h^{2} X+\phi\left(\nabla_{\xi} h\right) X
\end{aligned}
$$

for any vector fields $X, Y$ on $M$. 


\section{Weyl structures}

Now suppose that $(M, c)$ is a conformal manifold with conformal class $c$. A Weyl connection $D$ in $(M, c)$ is a torsion-free linear connection which preserves the conformal class $c$. For any metric $g$ in $c$ it carries a 1 -form $\theta$, called thee Lee form with respect to $g$, such that $D g=-2 \theta \otimes g$. It is related to the Levi-Civita connection $\nabla$ by the following relation:

$$
D_{X} Y=\nabla_{X} Y+\theta(X) Y+\theta(Y) X-g(X, Y) B
$$

for any vector fields $X, Y$, where $B$ is dual to $\theta$ with respect to $g$. A Weyl structure $W=$ $(D,[g])$ is said to be $c l o s e d$, resp. exact if its Lee form is closed, resp. exact with respect to any metric in $c$.

By (3.1), a straightforward computation implies the curvature tensor and Ricci tensor of the Weyl connection $D$ are as follows:

$$
\begin{aligned}
R^{D}(X, Y) Z= & R(X, Y) Z+\Sigma_{g}(X, Y) Z-\Sigma_{g}(Y, X) Z \\
\operatorname{Ric}^{D}(Y, Z)= & \operatorname{Ric}(Y, Z)-2 n\left(\nabla_{Z} \theta\right)(Y)+\left(\nabla_{Y} \theta\right)(Z) \\
& +(2 n-1) \theta(Z) \theta(Y)+\left(\delta \theta-(2 n-1)|\theta|^{2}\right) g(Y, Z),
\end{aligned}
$$

where

$$
\begin{aligned}
\Sigma_{g}(X, Y) Z= & \left(\nabla_{X} \theta\right)(Y) Z+\left(\nabla_{X} \theta\right)(Z) Y-g(Y, Z) \nabla_{X} B \\
& -g(Y, Z)|\theta|^{2} X-g(X, Z) \theta(Y) B+\theta(Y) \theta(Z) X
\end{aligned}
$$

for $X, Y, Z \in \mathfrak{X}(M)$ and $\delta \theta$ denotes the codifferential of $\theta$ with respect to $g$.

Moreover, the following characterization of closed Weyl connection was proved.

Proposition 3.1 [21] Let $(M, \xi, \eta, \phi, g)$ be a $(2 n+1)$-dimensional almost contact manifold. Then the Weyl structure $W=(D,[g])$ is closed if and only if $\eta\left(R^{D}(X, Y) \xi\right)=0$ for all vector fields $X, Y \in \mathfrak{X}(M)$.

It is well-known that for an almost contact manifold $M$ its tangent bundle $T M$ can be decomposed as $T M=\mathbb{R} \xi \oplus \mathcal{D}$, where $\mathcal{D}=\{X \in T M: \eta(X)=0\}$. Applying Proposition 3.1 , we immediately obtain the following result.

Proposition 3.2 Let $(M, \xi, \eta, \phi, g)$ be a $(2 n+1)$-dimensional almost contact manifold. If the Weyl structure $W=(D,[g])$ is closed, then either $B \in \mathbb{R} \xi$ or $B \in \mathcal{D}$.

Proof By Proposition 3.1, we obtain from (3.2) that for all vector fields $X, Y$,

$$
\begin{aligned}
& \left(\nabla_{X} \theta\right)(\xi) \eta(Y)-\eta(Y) \eta\left(\nabla_{X} B\right)-\eta(X) \theta(Y) \eta(B) \\
& \quad-\left[\left(\nabla_{Y} \theta\right)(\xi) \eta(X)-\eta(X) \eta\left(\nabla_{Y} B\right)-\eta(Y) \theta(X) \eta(B)\right]=0 .
\end{aligned}
$$

Here we have used the relation $\left(\nabla_{X} \theta\right) Y=\left(\nabla_{Y} \theta\right) X$ which follows from $d \theta=0$. Since

$$
\left(\nabla_{X} \theta\right)(\xi)=\nabla_{X}(g(B, \xi))-\theta\left(\nabla_{X} \xi\right)=g\left(\nabla_{X} B, \xi\right)=\eta\left(\nabla_{X} B\right),
$$

the above relation is simplified as

$$
[-\eta(X) \theta(Y)+\eta(Y) \theta(X)] \eta(B)=0 .
$$

Thus by taking $Y=\xi$, we see that $\theta(X) \eta(B)=0$ for all $X \in \mathcal{D}$, that means that either $B \in \mathcal{D}$ or $B \in \mathbb{R} \xi$. 
A Weyl structure $W=(D,[g])$ is called Einstein-Weyl if the trace-free component of the symmetric part of $\operatorname{Ric}^{D}$ is identically zero, namely there exists a smooth function $\Lambda$ such that the relation (1.1) holds. Thus it follows from (3.3) and (1.1) that

$$
\operatorname{Ric}(X, Y)-\frac{2 n-1}{2}\left(\left(\nabla_{X} \theta\right) Y+\left(\nabla_{Y} \theta\right) X\right)+(2 n-1) \theta(X) \theta(Y)=\sigma g(X, Y),
$$

where $\sigma=\delta \theta-(2 n-1)|\theta|^{2}+\frac{\Lambda}{2}$. Furthermore, if $M$ admits two Einstein-Weyl structures with $\theta$ and $-\theta$, then the following two equations hold for arbitrary vector fields $X, Y$ in $M$ (Higa [15]):

$$
\begin{aligned}
& \left(\nabla_{X} \theta\right) Y+\left(\nabla_{Y} \theta\right) X+\frac{2}{2 n+1} \delta \theta g(X, Y)=0, \\
& \operatorname{Ric}(X, Y)-\frac{r}{2 n+1} g(X, Y)=\frac{2 n-1}{2 n+1}|\theta|^{2} g(X, Y)-(2 n-1) \theta(X) \theta(Y) .
\end{aligned}
$$

Here $r$ denotes the scalar curvature of $M$.

Since the Weyl curvature tensor $R^{D}$ and the Weyl Ricci tensor $R i c^{D}$ of closed EinsteinWeyl structures defined on compact manifolds vanish identically (see [9]), from (3.2) and (3.3) we obtain

$$
\begin{aligned}
R(X, Y) Z= & \left\{\left(\nabla_{Y} \theta\right) Z-\theta(Y) \theta(Z)\right\} X-\left\{\left(\nabla_{X} \theta\right) Z-\theta(X) \theta(Z)\right\} Y \\
& +g(Y, Z)\left\{\left(\nabla_{X} B-\theta(X) B\right\}-g(X, Z)\left\{\left(\nabla_{Y} B-\theta(Y) B\right\}\right.\right. \\
& +|\theta|^{2}\{g(Y, Z) X-g(X, Z) Y\}, \\
\operatorname{Ric}(Y, Z)= & (2 n-1)\left(\nabla_{Y} \theta\right)(Z) \\
& -(2 n-1) \theta(Z) \theta(Y)-\left(\delta \theta-(2 n-1)|\theta|^{2}\right) g(Y, Z) .
\end{aligned}
$$

Moreover, using (3.8) we obtain

$$
(2 n-1) \nabla_{X} B=Q X+(2 n-1) \theta(X) B+\lambda X,
$$

where $\lambda=\delta \theta-(2 n-1)|\theta|^{2}$ and $Q$ is the Ricci operator defined by $\operatorname{Ric}(X, Y)=g(Q X, Y)$ for any vectors $X, Y$. Thus from (3.9), it is easy to yield

$$
\begin{aligned}
(2 n-1) R(X, Y) B= & \left(\nabla_{X} Q\right) Y-\left(\nabla_{Y} Q\right) X+\theta(Y) Q X \\
& -\theta(X) Q Y+\lambda[\theta(Y) X-\theta(X) Y] \\
& +(X \lambda) Y-(Y \lambda) X .
\end{aligned}
$$

Taking (3.9) into account, the formula (3.7) becomes

$$
\begin{aligned}
(2 n-1) R(X, Y) Z= & \{\operatorname{Ric}(Y, Z)-2(2 n-1) \theta(Y) \theta(Z)\} X \\
& -\{\operatorname{Ric}(X, Z)-2(2 n-1) \theta(X) \theta(Z)\} Y \\
& +g(Y, Z)\{Q X-2(2 n-1) \theta(X) B\} \\
& -g(X, Z)\{Q Y-2(2 n-1) \theta(Y) B\} \\
& +\left((2 n-1)|\theta|^{2}+2 \lambda\right)\{g(Y, Z) X-g(X, Z) Y\} .
\end{aligned}
$$


Furthermore, putting $Y=Z=\xi$ in (3.11) gives

$$
\begin{aligned}
(2 n-1) R(X, \xi) \xi= & \left\{\operatorname{Ric}(\xi, \xi)-2(2 n-1) \theta(\xi)^{2}\right\} X \\
& -\{\operatorname{Ric}(X, \xi)-2(2 n-1) \theta(X) \theta(\xi)\} \xi \\
& +\{q x-2(2 n-1) \theta(X) B\} \\
& -\eta(X)\{Q \xi-2(2 n-1) \theta(\xi) B\} \\
& +\left((2 n-1)|\theta|^{2}+2 \lambda\right)\{X-\eta(X) \xi\} .
\end{aligned}
$$

\section{Einstein-Weyl structures on almost cosymplectic $(\kappa, \mu)$-manifolds}

In this section we suppose that $\left(M^{2 n+1}, \phi, \xi, \eta, g\right)$ is an almost cosymplectic $(\kappa, \mu)$ manifold, namely the curvature tensor satisfies (1.2). By definition, the Eqs. (2.2)-(2.8) with $\alpha=0$ hold. Furthermore, the following relations are provided (see [5, Eqs. (3.22) and (3.23)]):

$$
\begin{aligned}
Q & =2 n \kappa \eta \otimes \xi+\mu h, \\
h^{2} & =\kappa \phi^{2} .
\end{aligned}
$$

Using (2.2), it follows from (4.1) that the scalar curvature $r=2 n \kappa$ and $Q \xi=2 n \kappa \xi$. By (4.2), we find easily that $\kappa \leq 0$ and $\kappa=0$ if and only if $M$ is a cosymplectic manifold, thus in the following we always suppose $\kappa<0$.

Theorem $4.1 A(2 n+1)$-dimensional almost $(\kappa, \mu)$-cosymplectic manifold admitting a closed Einstein-Weyl structure is an Einstein manifold or a cosymplectic manifold.

Proof By Proposition 3.2, we know that either $B \in \mathcal{D}$ or $B \in \mathbb{R} \xi$. Next we consider these two cases respectively.

We first assume that $\theta=f \eta$ for some function $f$. Since $d \theta=0$, by (2.3), Eq. (3.4) is rewritten as

$$
\begin{aligned}
& \operatorname{Ric}(X, Y)-(2 n-1)(X(f) \eta(Y)-f g(\phi h X, Y)) \\
& \quad+(2 n-1) f^{2} \eta(X) \eta(Y)=\sigma g(X, Y) .
\end{aligned}
$$

That is,

$$
Q X-(2 n-1) X(f) \xi+(2 n-1) f \phi h X+(2 n-1) f^{2} \eta(X) \xi=\sigma X .
$$

Applying (4.1) in the above formula gives

$$
2 n \kappa \eta(X) \xi+\mu h X-(2 n-1) X(f) \xi+(2 n-1) f \phi h X+(2 n-1) f^{2} \eta(X) \xi=\sigma X .
$$

Replacing $X$ by $h X$ and using (4.2), we have

$$
\sigma h X+(2 n-1) f \kappa \phi X=\kappa \mu \phi^{2} X-(2 n-1) h X(f) \xi .
$$

Moreover, by taking an inner product of the foregoing relation with $\phi X$ and contracting $X$ over the resulting equation, we get

$$
-\sigma \operatorname{trace}(\phi h)+2 n(2 n-1) f \kappa=0 .
$$

Thus the relation (2.7) shows that $f=0$ and $M$ is Einstein from (4.3). 
In the following we consider the case where $B \in \mathcal{D}$. In view of (4.1) and (2.3), the Eq. (3.4) with $Y=\xi$ becomes

$$
(2 n \kappa-\sigma) \eta(X)-(2 n-1) \theta(\phi h X)=0 .
$$

Putting $X=\xi$ implies $2 n \kappa=\sigma$, so we get $h B=0$ by the above formula. Furthermore it yields from (4.2) that $\kappa \phi^{2} B=0$, i.e., $B=0$.

On the other hand, contracting $X$ over (3.4) we have

$$
r-(2 n-1) \delta \theta+(2 n-1)|\theta|^{2}=(2 n+1) \sigma .
$$

Because the scalar curvature $r=2 n \kappa$, we derive

$$
\delta \theta-|\theta|^{2}=-\frac{4 n^{2} \kappa}{2 n-1} .
$$

It comes to a contradiction with $\kappa<0$, hence it is impossible.

Summing up the above discussion, we complete the proof.

If $M$ admits two Einstein-Weyl structures with $\theta$ and $-\theta$, we immediately prove the following result.

Theorem 4.2 A $(2 n+1)$-dimensional almost $(\kappa, \mu)$-cosymplectic manifold admitting two Einstein-Weyl structures with $\theta$ and $-\theta$ is either cosymplectic or Einstein.

Proof By (4.1), the formula (3.6) with $Y=\xi$ becomes

$$
\frac{4 n^{2} \kappa-(2 n-1)|\theta|^{2}}{2 n+1} \eta(X)=-(2 n-1) \theta(X) \theta(\xi),
$$

which shows that either $B \in \mathbb{R} \xi$ or $B \in \mathcal{D}$ by taking an arbitrary $X \in \mathcal{D}$.

If $B \in \mathbb{R} \xi$, we may set $B=f \xi$ for some smooth function $f$ on $M$. The Eq. (3.6) becomes

$$
\operatorname{Ric}(X, Y)=\frac{r+(2 n-1) f^{2}}{2 n+1} g(X, Y)-(2 n-1) f^{2} \eta(X) \eta(Y)
$$

Furthermore, in terms of (3.5), we get

$$
X(f) \eta(Y)-2 f g(\phi h X, Y)+Y(f) \eta(X)+\frac{2}{2 n+1} \xi(f) g(X, Y)=0 .
$$

Replacing $X$ by $h X$ and $Y$ by $\phi X$ and contracting $X$ over the resulting equation, we can prove that $f=0$ or $h=0$. Therefore $M$ is an Einstein manifold or a cosymplectic manifold by (4.5).

For the case where $B \in \mathcal{D}$, we derive from (4.4) that $4 n^{2} \kappa=(2 n-1)|\theta|^{2}$. Since $\kappa<0$, it leads to a contradiction.

\section{Einstein-Weyl structures on almost $\alpha$-cosymplectic manifolds}

In this section we study an almost $\alpha$-cosymplectic manifold admitting Einstein-Weyl structures. First we consider the case of three dimension.

Theorem 5.1 Let $\left(M^{3}, \Phi, \xi, \eta, g\right)$ be a compact almost $\alpha$-cosymplectic manifold. Suppose that $M$ admits a closed Einstein-Weyl structure. Then $M$ is Ricci-flat. 
Proof As before, by Proposition 3.2, $B \in \mathcal{D}$ or $\theta=f \eta$ where $f=\theta(\xi)$. Next we divide into two cases to discuss.

Case I. First we set $\theta=f \eta$ for some function $f$. By (2.3), we have

$$
\nabla_{X} B=X(f) \xi-f\left(\alpha \phi^{2} X+\phi h X\right) .
$$

Since $d \theta=0$, i.e., $g\left(\nabla_{X} B, Y\right)=g\left(X, \nabla_{Y} B\right)$ for all $X, Y \in \mathfrak{X}(M)$, we get $X(f) \eta(Y)=$ $Y(f) \eta(X)$. That means that the gradient vector field $D f=\xi(f) \xi$. Applying Poincare lemma $d^{2}=0$, we obtain $g\left(\nabla_{X} D f, Y\right)=g\left(X, \nabla_{Y} D f\right)$ for all $X, Y$, thus $\xi(\xi(f)) \eta(X)=X(\xi(f))$ by (2.2). Using (5.1), the formula (3.9) becomes

$$
Q X=X(f) \xi-f\left(\alpha \phi^{2} X+\phi h X\right)-f^{2} \eta(X) \xi-\lambda X .
$$

Furthermore, the scalar curvature $r=\xi(f)+2 \alpha f-f^{2}-3 \lambda$. In terms of (5.1) and using (2.7), we compute $\lambda=\delta \theta-|\theta|^{2}=\xi(f)+2 \alpha f-f^{2}$, so we find $r=-2 \lambda$.

On the other hand, it is well known that the curvature tensor of a 3-dimensional Riemannian manifold is given by

$$
\begin{aligned}
R(X, Y) Z= & g(Y, Z) Q X-g(X, Z) Q Y+g(Q Y, Z) X-g(Q X, Z) Y \\
& -\frac{r}{2}\{g(Y, Z) X-g(X, Z) Y\} .
\end{aligned}
$$

Hence substituting (5.2) into (5.3) yields

$$
\begin{aligned}
R(X, Y) Z= & g(Y, Z)\left[X(f) \xi-f\left(\alpha \phi^{2} X+\phi h X\right)-f^{2} \eta(X) \xi\right] \\
& -g(X, Z)\left[Y(f) \xi-f\left(\alpha \phi^{2} Y+\phi h Y\right)-f^{2} \eta(Y) \xi\right] \\
& +g\left(Y(f) \xi-f\left(\alpha \phi^{2} Y+\phi h Y\right)-f^{2} \eta(Y) \xi-\lambda Y, Z\right) X \\
& -g\left(X(f) \xi-f\left(\alpha \phi^{2} X+\phi h X\right)-f^{2} \eta(X) \xi-\lambda X, Z\right) Y .
\end{aligned}
$$

Putting $Y=Z=\xi$ gives

$$
\begin{aligned}
R(X, \xi) \xi= & -f\left(\alpha \phi^{2} X+\phi h X\right)+\left(\xi(f)-f^{2}-\lambda\right) X \\
& -\left(X(f)-f^{2} \eta(X)-\lambda \eta(X)\right) \xi .
\end{aligned}
$$

Connecting this with (2.8) implies

$$
\begin{aligned}
& \alpha^{2} \phi^{2} X+2 \alpha \phi h X-h^{2} X+\phi\left(\nabla_{\xi} h\right) X \\
& \quad=-f\left(\alpha \phi^{2} X+\phi h X\right)-2 \alpha f X-(X(f)-(\xi(f)+2 \alpha f) \eta(X)) \xi
\end{aligned}
$$

On the other and, differentiating (5.2) along $Y$ and using (2.3), we conclude

$$
\begin{aligned}
\left(\nabla_{Y} Q\right) X= & Y(\xi(f)) \eta(X) \xi+\xi(f) g\left(\nabla_{Y} \xi, X\right) \xi+X(f) \nabla_{Y} \xi \\
& -f\left(\alpha\left(\nabla_{Y} \phi^{2}\right) X+\left(\nabla_{Y} \phi\right) h X+\phi\left(\nabla_{Y} h\right) X\right) \\
& -2 f Y(f) \eta(X) \xi-f^{2} g\left(\nabla_{Y} \xi, X\right) \xi \\
& -f^{2} \eta(X) \nabla_{Y} \xi-Y(\lambda) X .
\end{aligned}
$$


Thus the Eq. (3.10) becomes

$$
\begin{aligned}
R(X, Y) B= & Y(\xi(f)) \eta(X) \xi+\xi(f) g\left(\nabla_{Y} \xi, X\right) \xi+X(f) \nabla_{Y} \xi \\
& -f\left(\alpha\left(\nabla_{Y} \phi^{2}\right) X+\left(\nabla_{Y} \phi\right) h X+\phi\left(\nabla_{Y} h\right) X\right) \\
& -3 f Y(f) \eta(X) \xi-f^{2} g\left(\nabla_{Y} \xi, X\right) \xi-2 f^{2} \eta(X) \nabla_{Y} \xi \\
& -\left[X(\xi(f)) \eta(Y) \xi+\xi(f) g\left(\nabla_{X} \xi, Y\right) \xi+Y(f) \nabla_{X} \xi\right. \\
& -f\left(\alpha\left(\nabla_{X} \phi^{2}\right) Y+\left(\nabla_{X} \phi\right) h Y+\phi\left(\nabla_{X} h\right) Y\right) \\
& \left.-3 f X(f) \eta(Y) \xi-f^{2} g\left(\nabla_{X} \xi, Y\right) \xi-2 f^{2} \eta(Y) \nabla_{X} \xi\right] \\
& +2(X \lambda) Y-2(Y \lambda) X .
\end{aligned}
$$

By comparing (5.6) and (5.4) with $Z=B$, we have

$$
\begin{aligned}
& Y(\xi(f)) \eta(X) \xi+\xi(f) g\left(\nabla_{Y} \xi, X\right) \xi+X(f) \nabla_{Y} \xi \\
& \quad-f\left(\alpha\left(\nabla_{Y} \phi^{2}\right) X+\left(\nabla_{Y} \phi\right) h X+\phi\left(\nabla_{Y} h\right) X\right) \\
& \quad-2 f Y(f) \eta(X) \xi-f^{2} g\left(\nabla_{Y} \xi, X\right) \xi-f^{2} \eta(X) \nabla_{Y} \xi \\
& \quad-\left[X(\xi(f)) \eta(Y) \xi+\xi(f) g\left(\nabla_{X} \xi, Y\right) \xi+Y(f) \nabla_{X} \xi\right. \\
& \quad-f\left(\alpha\left(\nabla_{X} \phi^{2}\right) Y+\left(\nabla_{X} \phi\right) h Y+\phi\left(\nabla_{X} h\right) Y\right) \\
&\left.\quad-2 f X(f) \eta(Y) \xi-f^{2} g\left(\nabla_{X} \xi, Y\right) \xi-f^{2} \eta(Y) \nabla_{X} \xi\right]+2(X \lambda) Y-2(Y \lambda) X . \\
&= f\left(Y(f)-f^{2} \eta(Y)-\lambda \eta(Y)\right) X-f\left(X(f)-f^{2} \eta(X)-\lambda \eta(X)\right) Y .
\end{aligned}
$$

Now let us put $Y=\xi$, then the above formula is simplified as

$$
\begin{aligned}
- & f\left(\phi\left(\nabla_{\xi} h\right) X\right)-2 f \xi(f) \eta(X) \xi \\
- & {\left[\xi(f) \nabla_{X} \xi+f\left(\alpha^{2} \phi^{2} X+2 \alpha \phi h X-h^{2} X\right)\right.} \\
& \left.-f X(f) \xi-f^{2} \nabla_{X} \xi\right]+2(X \lambda) \xi-2 \xi(\lambda) X . \\
= & f\left(\xi(f)-f^{2}-\lambda\right) X+f \eta(X)\left(f^{2}+\lambda\right) \xi .
\end{aligned}
$$

Furthermore, by (5.5), the above formula becomes

$$
\begin{aligned}
- & 2 f \xi(f) \eta(X) \xi-\xi(f) \nabla_{X} \xi+2 f X(f) \xi+2 X(\lambda) \xi-2 \xi(\lambda) X \\
& =-4 f^{2} \alpha X+2 f \eta(X)\left(f^{2}+\lambda\right) \xi .
\end{aligned}
$$

Take $X=e_{1} \in \mathcal{D}$ such that $h e_{1}=v e_{1}$ with $v$ being a non-zero function, so the above relation becomes

$$
-\xi(f) \nabla_{e_{1}} \xi+2 e_{1}(\lambda) \xi-2 \xi(\lambda) e_{1}=-4 f^{2} \alpha e_{1} .
$$

From (2.3), we obtain

$$
\left\{\begin{array}{l}
\xi(f) v=0, \\
\xi(f) \alpha+2 \xi(\lambda)=4 f^{2} \alpha .
\end{array}\right.
$$


Since $v \neq 0$ and $\lambda=\xi(f)+2 \alpha f-f^{2}$, the foregoing relations show that $f=0$, so $M$ is Ricci-flat by (5.2).

Case II. When $B \in \mathcal{D}$, from (3.12) and (5.3) with $n=1$, we follow

$$
0=-2 \theta(X) B+\left(|\theta|^{2}+2 \lambda+\frac{r}{2}\right)\{X-\eta(X) \xi\} .
$$

When $X=B$, the above relation becomes

$$
\left(|\theta|^{2}-2 \lambda-\frac{r}{2}\right) B=0 .
$$

Thus $|\theta|^{2}-2 \lambda-\frac{r}{2}=0$ or $B=0$. If $|\theta|^{2}-2 \lambda-\frac{r}{2}=0$, the formula (5.7) becomes

$$
0=-\theta(X) B+|\theta|^{2}\{X-\eta(X) \xi\} .
$$

Contracting $X$ over this equation, we also get $|\theta|^{2}=0$. By (3.8), thus $M$ is Ricci-flat.

Next we consider the case where $M$ admits two Einstein-Weyl structures $\pm \theta$ and obtain the following result.

Theorem 5.2 Let $\left(M^{2 n+1}, \phi, \xi, \eta, g\right)$ be an almost $\alpha$-cosymplectic manifold. Suppose that $M$ admits two Einstein-Weyl structures with $\pm \theta$. If the Ricci tensor is commuting, i.e., $\phi Q=Q \phi$, then $M$ is either an Einstein manifold, or an $\alpha$-cosymplectic manifold.

Proof By (3.6), the Ricci operator is expressed as

$$
Q X=\left(\frac{2 n-1}{2 n+1}|\theta|^{2}+\frac{r}{2 n+1}\right) X-(2 n-1) \theta(X) B .
$$

Since the Ricci tensor is commuting,

$$
\theta(X) \phi B=\theta(\phi X) B .
$$

Taking $X=B$ gives $\phi B=0$ or $B=0$. Thus we know that $B=f \xi$, where $f=\theta(\xi)$. In terms of (3.5), we get

$$
X(f) \eta(Y)-2 f \alpha g\left(\phi^{2} X, Y\right)-2 f g(\phi h X, Y)+Y(f) \eta(X)+\frac{2}{2 n+1} \delta \theta g(X, Y)=0 .
$$

As the proof of Theorem 4.2, replacing $X$ by $h X$ and $Y$ by $\phi X$, contracting $X$ over the resulting equation and using (2.7), we obtain $f=0$ or $h=0$. Therefore we complete the proof by (5.8).

Corollary 5.3 Let $\left(M^{2 n+1}, \phi, \xi, \eta, g\right)$ be an almost $\alpha$-cosymplectic manifold. Suppose that $M$ admits two Einstein-Weyl structures with $\pm \theta= \pm f \eta$ for some function $f$, then $M$ either is an Einstein manifold, or an $\alpha$-cosymplectic manifold.

\section{Einstein-Weyl structures on $K$-cosymplectic manifolds}

Let $M$ be a $(2 n+1)$-dimensional almost cosymplectic manifold defined in Sect. 2, namely the 1 -form $\eta$ and the fundamental form $\omega$ are closed and satisfy $\eta \wedge \omega^{n} \neq 0$ at every point of $M$.

Definition 6.1 [2] An almost cosymplectic manifold $(M, \phi, \xi, \eta, g)$ is called a $K$-cosymplectic manifold if the Reeb vector field $\xi$ is Killing. 
For a $K$-cosymplectic manifold $(M, \phi, \xi, \eta, g)$, by Theorem 3.11 in [5] we know

$$
\nabla \xi=\nabla \eta=0 .
$$

Moreover, it follows from Theorem 3.29 in [5] that

$$
R(X, Y) \xi=0 \text { for all } X, Y \in \mathfrak{X}(M) .
$$

That shows that $Q \xi=0$.

In the following we suppose that $M$ admits a closed Einstein-Weyl structure, hence either $B \in \mathbb{R} \xi$ or $B \in \mathcal{D}$ by Proposition 3.2.

If $B \in \mathbb{R} \xi$, we set $\theta=f \eta$ for some smooth function $f$ on $M$. Then the Ricci tensor (3.8) becomes

$$
\operatorname{Ric}(Y, X)=(2 n-1) X(f) \eta(Y)-(2 n-1) f^{2} \eta(X) \eta(Y)-\lambda g(Y, X) .
$$

Using (6.1), the formula (3.12) yields

$$
\begin{aligned}
Q X & =-\left((2 n-1)|\theta|^{2}+2 \lambda-2(2 n-1) f^{2}\right)\{X-\eta(X) \xi\} \\
& =-\left(2 \lambda-(2 n-1) f^{2}\right)\{X-\eta(X) \xi\} .
\end{aligned}
$$

Combining with (6.2), we conclude that

$$
-\left(\lambda-(2 n-1) f^{2}\right) X=(2 n-1) X(f) \xi-2 \lambda \eta(X) \xi .
$$

Letting $X \in \mathcal{D}$ we find $X(f)=0$ and $\lambda=(2 n-1) f^{2}$. From this we see that $D f=\xi(f) \xi$ with $\xi(f)=\frac{2 \lambda}{2 n-1}$. On the other hand, since $\lambda=\delta \theta-(2 n-1)|\theta|^{2}, \delta \theta=2 \lambda$. Because $\xi$ is Killing, $\delta \theta=\xi(f)$, which yields $\xi(f)=0$, and further $f=0$. That means that $M$ is Ricci-flat, thus it is cosymplectic in terms of Corollary 3.35 in [5].

Next we assume $B \in \mathcal{D}$, then $\theta(\xi)=0$. From (3.12) and (6.1), the Ricci operator $Q$ is expressed as

$$
Q X=2(2 n-1) \theta(X) B-\left((2 n-1)|\theta|^{2}+2 \lambda\right)\{X-\eta(X) \xi\} .
$$

Since $\xi$ is Killing and $Q \xi=0$, by (3.8) with $Y=Z=\xi$, we see that $\lambda=0$, i.e., $\delta \theta=(2 n-1)|\theta|^{2}$. On the other hand, via (6.3) and (3.9), we have

$$
\nabla_{X} B=3 \theta(X) B-|\theta|^{2}\{X-\eta(X) \xi\} .
$$

Contracting $X$ over the foregoing equation gives $\delta \theta=(3-2 n)|\theta|^{2}$. Hence we get $\theta=0$.

Summing up the above discussion, we actually proved the following conclusion.

Theorem 6.2 Let $(M, \phi, \xi, \eta, g)$ be a compact $(2 n+1)$-dimensional $K$-cosymplectic manifold. Suppose that $M$ admits a closed Einstein-Weyl structure. Then $M$ is cosymplectic.

For a $K$-cosymplectic manifold with two Einstein-Weyl structures with $\pm \theta$, we also have the following conclusion.

Theorem 6.3 Let $(M, \phi, \xi, \eta, g)$ be a $(2 n+1)$-dimensional, $2 n+1 \geq 3, K$-cosymplectic manifold. Suppose that $M$ admits two Einstein-Weyl structures with $\pm \theta$. Then either $M$ is Ricci-flat, or the scalar curvature is non-positive and invariant along the Reeb vector field $\xi$. 
Proof Since $Q \xi=0$, it follows from (3.6) that

$$
\left[-\frac{r}{2 n+1}-\frac{2 n-1}{2 n+1}|\theta|^{2}\right] \eta(X)=(2 n-1) \theta(X) \theta(\xi) .
$$

By taking $X \in \mathcal{D}$, we see that $B \in \mathbb{R} \xi$ or $B \in \mathcal{D}$. When $B \in \mathbb{R} \xi$, as before we set $B=f \xi$, then $\delta \theta=\xi(f)$, so it follows from (3.5)

$$
X(f) \eta(Y)+Y(f) \eta(X)+\frac{2 \xi(f)}{2 n+1} g(X, Y)=0 .
$$

Replacing $X$ and $Y$ by $\phi X$, we get $\xi(f)=0$. Further the above relation implies $f=0$. Therefore the Eq. (6.4) yields $r=0$ and $M$ is Ricci-flat from (3.6).

If $B \in \mathcal{D}$, (6.4) implies that $r=-(2 n-1)|\theta|^{2}$, and further $Q X=-(2 n-1) \theta(X) B$ by (3.6). So $Q B=r B$. Since $B \in \mathcal{D}$ and $\xi$ is Killing, taking $X=\xi$ and $Y=B$ in (3.5) yields $\left(\nabla_{\xi} \theta\right) B=0$. Thus we find $\xi(r)=-2(2 n-1)\left(\nabla_{\xi} \theta\right) B=0$.

Since any compact Ricci-flat almost cosymplectic manifold is cosymplectic (see [5, Corollary 3.35$]$ ), we conclude immediately from the previous theorem the following corollary.

Corollary 6.4 A compact $K$-cosymplectic manifold admitting two Einstein-Weyl structures with $\pm \theta= \pm f \eta$ for some function $f$ is cosymplectic.

Acknowledgements The author would like to thank the referee for the comments and valuable suggestions.

Open Access This article is distributed under the terms of the Creative Commons Attribution 4.0 International License (http://creativecommons.org/licenses/by/4.0/), which permits unrestricted use, distribution, and reproduction in any medium, provided you give appropriate credit to the original author(s) and the source, provide a link to the Creative Commons license, and indicate if changes were made.

\section{References}

1. G. Bazzoni, O. Goertsches, K-Cosymplectic manifolds. Ann. Glob. Anal. Geom. 47, 1-32 (2014)

2. C.P. Boyer, K. Galicki, Einstein manifolds and contact geometry. Proc. Am. Math. Soc. 129, 2419-2430 (2001)

3. C.P. Boyer, K. Galicki, On Sasakian-Einstein geometry. Int. J. Math. 11(7), 873-909 (2000)

4. B. Cappelletti-Montano, A.M. Pastore, Einstein-like conditions and cosymplectic geometry. J. Adv. Math. Stud. 3(2), 27-40 (2010)

5. B. Cappelletti-Montano, A. De Nicola, A.I. Yudin, A survey on cosymplectic geometry. Rev. Math. Phys. 25, $1343002(2013)$

6. T.W. Kim, H.K. Pak, Canonical foliations of certain classes of almost contact metric structres. Acta Math. Sinica Eng. Ser. Aug. 21, 841-846 (2005)

7. P. Dacko, On almost cosymplectic manifolds with the structure vector field $\xi$ belonging to the $\kappa$-nullity distribution. Balkan J. Geom. Appl. 5(2), 47-60 (2000)

8. H. Endo, Non-existence of almost cosymplectic manifolds satisfying a certain condition. Tensor (N.S.) 63(3), 272-284 (2002)

9. P. Gauduchon, Structures de Weyl-Einstein, espaces de twisteurs et variétés de type $S^{1} \times S^{3}$. J. Reine Angew. Math. 469, 1-50 (1995)

10. P. Gauduchon, A. Moroianu, Weyl-Einstein structures on K-contact manifolds. Geom. Dedicata. 189(1), 177-184 (2017)

11. A. Ghosh, Einstein-Weyl structures on contact metric manifolds. Ann. Glob. Anal. Geom. 35(4), 431-441 (2009)

12. A. Ghosh, On the closed Einstein-Weyl structure and compact K-contact manifold. Bull. Korean Math. Soc. 53(6), 1869-1878 (2016)

13. S.I. Goldberg, Integrability of almost Kaehler manifolds. Proc. Am. Math. Soc. 21, 96-100 (1969)

14. S.I. Goldberg, K. Yano, Integrability of almost cosymplectic structure. Pac. J. Math. 31, 373-382 (1969) 
15. T. Higa, Weyl manifolds and Einstein-Weyl manifolds. Comment. Math. Univ. St. Paul. 42, 143-160 (1993)

16. N.J. Hitchin, Monopoles and geodesies. Commun. Math. Phys. 83, 579-602 (1982)

17. N.J. Hitchin, Complex manifolds and Einstein equations, in Twistor Geometry and Non-linear Systems, Lecture Notes in Mathematics, vol. 970 (Springer, Berlin, 1982), p. 79-99

18. K. Kenmotsu, A class of almost contact Riemannian manifolds. Tôhoku Math. J. 24, 93-103 (1972)

19. P. Matzeu, Some examples of Einstein-Weyl structures on almost contact manifolds. Class. Quantum Gravity 17(24), 5079-5087 (2000)

20. P. Matzeu, Almost contact Einstein-Weyl structures. Manuscr. Math. 108(3), 275-288 (2002)

21. P. Matzeu, Closed Einstein-Weyl structures on compact Sasakian and cosymplectic manifolds. Proc. Edinb. Math. Soc. 54(1), 149-160 (2011)

22. F. Narita, Einstein-Weyl structures on almost contact metric manifolds. Tsukuba J. Math. 22(1), 87-98 (1998)

23. Z. Olszak, On almost cosymplectic manifolds. Kodai Math. J. 4, 239-250 (1981)

24. H. Öztürk, N. Aktan, C. Murathan, Almost $\alpha$-cosymplectic $(\kappa, \mu, v)$-spaces. arxiv:1007.0527v1

25. K. Sekigawa, On some compact Einstein almost Kaehler manifolds. J. Math. Soc. Jpn. 39, 677-684 (1983)

26. C. Udriste, Geometric dynamics. South. Asian Bull. Math. 24, 313-22 (2000)

27. H. Weyl, Raum Zeit Materie J (Springer, Berlin, 1923)

Publisher's Note Springer Nature remains neutral with regard to jurisdictional claims in published maps and institutional affiliations. 